\title{
A Stochastic Switched Epidemic Model with Two Epidemic Diseases
}

\author{
Amine El Koufi (D), Abdelkrim Bennar, and Noura Yousfi (iD \\ Laboratory of Analysis, Modeling and Simulation (LAMS), Faculty of Sciences Ben M'sik, Hassan II University, \\ P.O. Box 7955 Sidi Othman, Casablanca, Morocco
}

Correspondence should be addressed to Amine El Koufi; elkoufiamine1@gmail.com

Received 4 February 2021; Revised 2 March 2021; Accepted 5 March 2021; Published 25 March 2021

Academic Editor: Eric Campos

Copyright $(92021$ Amine El Koufi et al. This is an open access article distributed under the Creative Commons Attribution License, which permits unrestricted use, distribution, and reproduction in any medium, provided the original work is properly cited.

In this paper, we study a stochastic epidemic model with double epidemics which includes white noise and telegraph noise modeled by Markovian switching. Sufficient conditions for the extinction and persistence of the diseases are established. In the end, some numerical simulations are presented to demonstrate our analytical results.

\section{Introduction}

Compartmental models represent an important mathematical tool to describe the spread of infectious diseases [1-6]. A mathematical model that describes the spread of infectious diseases currently known as the SIR model divides the population into three classes, the sensitive $S$, infectious $I$, and recovered $R$, and was firstly developed by Kermack and Mckendrick [7] in 1927. To take into account the case of recovered individuals who lose their immunity and return to the susceptible compartment, we use SIRS epidemic models investigated by many scientists (see, for example, [8-10]). In classic SIRS epidemic models, the disease caused by one virus but in the real world, there are certain diseases to which the population is infected with two or more viruses (see [11-14]). Zhao et al. [15] established the following SIRS model describing the dynamics of two viruses (see Figure 1):

$$
\left\{\begin{array}{l}
S^{\prime}(t)=\mu-\beta_{1} S(t) I_{1}(t)-\beta_{2} S(t) I_{2}(t)-\mu S(t)+\delta R(t) \\
I_{1}^{\prime}(t)=\beta_{1} S(t) I_{1}(t)-\left(\mu+\gamma_{1}\right) I_{1}(t) \\
I_{2}^{\prime}(t)=\beta_{2} S(t) I_{2}(t)-\left(\mu+\gamma_{2}\right) I_{2}(t) \\
R^{\prime}(t)=\gamma_{1} I_{1}(t)+\gamma_{2} I_{2}(t)-(\mu+\delta) R(t)
\end{array}\right.
$$

where $S(t)$ denotes the number of susceptible individuals at time $t, I_{1}(t)$ and $I_{2}(t)$ are, respectively, the numbers of infected individuals with virus $A$ and virus $B, R(t)$ denotes the number of removed individuals at time, the parameters $\beta_{1}$ and $\beta_{2}$ are the disease transmission coefficient with virus $A$ and virus $B$, respectively, $\mu$ represents the birth rate of the population and the natural death rate of the population, $\gamma_{1}$ and $\gamma_{2}$ are the recovery rates of infectious individuals with virus $A$ and with virus $B$, and $\delta$ is the lost immunity rate.

However, the biological systems are necessarily touched via the environmental noise, which can present an additional degree of realism compared with their corresponding deterministic models. Thus, it is an important component in an ecosystem. Consequently, many works existing in the literature studied the effects of white noise on epidemic models (see, for example, [16-18]). El Fatini et al. [19] studied a stochastic epidemic model. They assumed that environmental perturbations are of the white noise type. They showed the existence and the uniqueness of the solution of their proposed model. They established sufficient conditions for extinction by constructing some suitable Lyapunov function. And they showed sufficient conditions for the persistence of the disease under some conditions on parameters of the model. Liu et al. [20] investigated the dynamical behavior of a higher-order stochastically perturbed SIRI epidemic model with relapse and media coverage. They showed sufficient measures for the existence and uniqueness of an ergodic stationary distribution of their stochastic model by constructing a suitable Lyapunov function. And 


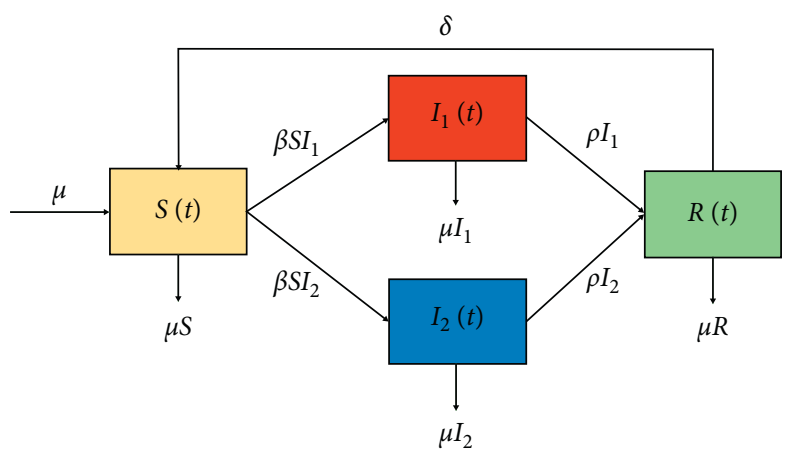

FIgURE 1: The compartmental diagram for the SIRS model with two epidemic diseases.

they obtained sufficient conditions for the elimination of the infectious disease. Tang et al. [21] investigated the threshold of a class of the stochastic SIRS epidemic model with with nonlinear incidence, which determines the extinction and persistence of the disease. In [22], Qi et al. investigated the dynamics of a nonautonomous stochastic SIS epidemic model with nonlinear incidence rate and double epidemic. Rajasekar and Pitchaimani [23] have formulated the following stochastic SIRS model with two viruses:

$$
\left\{\begin{aligned}
\mathrm{d} S(t) & =\left[\mu-\beta_{1} S(t) I_{1}(t)-\beta_{2} S(t) I_{2}(t)-\mu S(t)+\delta R(t)\right] \mathrm{d} t+\sigma_{1} S(t) \mathrm{d} B_{1}(t) \\
\mathrm{d} I_{1}(t) & =\left[\beta_{1} S(t) I_{1}(t)-\left(\mu+\gamma_{1}\right) I_{1}(t)\right] \mathrm{d} t+\sigma_{2} I_{1}(t) \mathrm{d} B_{2}(t), \\
\mathrm{d} I_{2}(t) & =\left[\beta_{2} S(t) I_{2}(t)-\left(\mu+\gamma_{2}\right) I_{2}(t)\right] \mathrm{d} t+\sigma_{3} I_{2}(t) \mathrm{d} B_{3}(t), \\
\mathrm{d} R(t) & =\left[\gamma_{1} I_{1}(t)+\gamma_{2} I_{2}(t)-(\mu+\delta) R(t)\right] \mathrm{d} t+\sigma_{4} R(t) \mathrm{d} B_{4}(t)
\end{aligned}\right.
$$

where $B_{i}(t)$ for $i=1,2,3,4$ are independent standard Brownian motions which are defined on a complete probability space $(\Omega, \mathscr{F}, \mathbb{P})$ with filtration $\left\{\mathscr{F}_{t}\right\}_{t \geq 0}$ satisfying the usual conditions and $\sigma_{i}^{2}$ for $i=1,2,3,4$ denote the intensity of the white noise.

On the other hand, the white noise cannot express the phenomena that the population may submit sudden catastrophic collisions in nature. The telegraph noise can be illustrated as a switching between two or more regimes of environment, which differ by factors such as nutrition or socio-cultural factors [24-26]. In fact, switching between environmental regimes is frequently memoryless and the waiting time for the next switching follows the exponential distribution [27]. So, the regime switching can be modeled by a continuous-time Markov chain $\{r(t)\}_{t \geq 0}$ taking values in a finite-state space $\mathbb{S}=\{1, \ldots, m\}$. Many works have introduced Markov process into their models. For example, in [28], Zhang and Peng addressed a stochastic cholera epidemic model with vaccination. They presented the threshold of a stochastic cholera epidemic model which determines the extinction of the disease and the existence of a unique ergodic stationary distribution. Wang et al. [29] discussed the asymptotic properties of the solution of a stochastic SIRS epidemic model with Beddington-DeAngelis incidence rate and Markovian switching. They proved that the proposed model is stochastically asymptotically stable in the large. In addition, they presented threshold values and conditions which determine disease extinction and persistence. In [30], Li et al. proposed a stochastic SIRS model with vaccination and regime switching. They proved sufficient conditions for the extinction and the existence of the stationary distribution of the population. Motivated by the above facts, in this work, we will initially include telegraph noise into stochastic model (1) to obtain a more realistic model. Then, the following stochastic white and telegraphic noise perturbations on the diseases transmission coefficient are assumed:

$$
\begin{aligned}
& \beta_{1} \mathrm{~d} t \longrightarrow \beta_{1}(r(t)) \mathrm{d} t+\sigma_{1}(r(t)) \mathrm{d} B_{1}(t), \\
& \beta_{2} \mathrm{~d} t \longrightarrow \beta_{2}(r(t)) \mathrm{d} t+\sigma_{2}(r(t)) \mathrm{d} B_{2}(t) .
\end{aligned}
$$

Then, the stochastic SIRS epidemic model with two viruses (1) can be expressed as the following stochastic system under regime switching: 


$$
\left\{\begin{aligned}
\mathrm{d} S(t)= & {\left[\mu(r(t))-\beta_{1}(r(t)) S(t) I_{1}(t)-\beta_{2}(r(t)) S(t) I_{2}(t)-\mu(r(t)) S(t)\right.} \\
& +\delta(r(t)) R(t)] \mathrm{d} t-\sigma_{1}(r(t)) S(t) I_{1}(t) \mathrm{d} B_{1}(t)-\sigma_{2}(r(t)) S(t) I_{2}(t) \mathrm{d} B_{2}(t), \\
\mathrm{d} I_{1}(t)= & {\left[\beta_{1}(r(t))(t) I_{1}(t)-\left(\mu(r(t))+\gamma_{1}(r(t))\right) I_{1}(t)\right] \mathrm{d} t } \\
& +\sigma_{1}(r(t)) S(t) I_{1}(t) \mathrm{d} B_{1}(t), \\
\mathrm{d} I_{2}(t)= & {\left[\beta_{2}(r(t)) S(t) I_{2}(t)-\left(\mu(r(t))+\gamma_{2}(r(t))\right) I_{2}(t)\right] \mathrm{d} t } \\
& +\sigma_{2}(r(t)) S(t) I_{2}(t) \mathrm{d} B_{2}(t), \\
\mathrm{d} R(t)= & \gamma_{1}(r(t)) I_{1}(t)+\gamma_{2}(r(t)) I_{2}(t)-(\mu(r(t))+\delta(r(t))) R(t) .
\end{aligned}\right.
$$

This article proposes a stochastic SIRS epidemic model with regime switching and double epidemic hypothesis. Our main purpose is to investigate the effect of the white and telegraph noises on the spread dynamics of the disease in the population. The rest of the paper is as follows. In Section 3, we show the existence and uniqueness of a global positive solution to system (4). In Sections 4 and 5, we study the existence of a stochastic threshold for the extinction and the persistence in mean of the disease. In last section, we present some numerical simulations to demonstrate our main theoretical results.

\section{Existence and Uniqueness of the Nonnegative Solution}

Throughout this paper, we let $\left(\Omega, \mathscr{F}_{,}\left\{\mathscr{F}_{t}\right\}_{t \geq 0}, \mathbb{P}\right)$ be a complete probability space with a filtration $\left\{\mathscr{F}_{t}\right\}_{t \geq 0}$ satisfying the usual conditions (i.e., it is increasing and right continuous while $\mathscr{F}_{0}$ contains all $\mathbb{P}$-null sets). Let $\{r(t)\}_{t \geq 0}$ be a right-continuous Markov chain on the probability space $\left(\Omega, \mathscr{F},\left\{\mathscr{F}_{t}\right\}_{t \geq 0}, \mathbb{P}\right)$ taking values in a finite-state space $\mathbb{S}=$ $\{1,2, \ldots, m\}$ with the generator $\Phi=\left(\phi_{u v}\right)_{1 \leq u, v \leq N}$ given, for $\delta>0$, by

$$
P(r(t+\delta)=v \mid r(t)=u)= \begin{cases}\phi_{u v} \delta+o(\delta), & \text { if } u \neq v \\ 1+\phi_{u u} \delta+o(\delta), & \text { if } u=v\end{cases}
$$

Here, $\phi_{u v}$ is the transition rate from $u$ to $v$ and $\phi_{u v} \geq 0$ if $u \neq v$, while

$$
\phi_{u u}=-\sum_{u \neq v} \phi_{u v}
$$

Suppose that the Markov chain $r(t)$ is independent of the Brownian motion $B(\cdot)$ and it is irreducible. Under this condition, the Markov chain has a unique stationary distribution $\pi=\left(\pi_{1}, \ldots, \pi_{N}\right)$, which can be determined by solving the linear equation $\pi \Phi=0$, subject to $\sum_{i=1}^{N} \pi_{i}=1$, and $\pi_{i}>0, \forall i \in \mathbb{S}$. Thereafter, for any vector $h=(h(1), \ldots, h(N))^{T}$, let $\widehat{h}=\min _{i \in \mathbb{M}}\{h(i)\} \quad$ and $\check{h}=\max _{i \in \mathbb{M}}\{h(i)\}$.

We consider the following stochastic system:

$$
\mathrm{d} \vartheta(t)=f(t, \vartheta(t), r(t)) \mathrm{d} t+g(t, \vartheta(t), r(t)) \mathrm{d} B(t),
$$

where $B(t)$ is a d-dimensional standard Wiener process defined on a complete probability space $\left(\Omega, \mathscr{F},\left\{\mathscr{F}_{t}\right\}_{t \geq 0}, \mathbb{P}\right)$.
Denoted by $C^{1,2}\left(\mathbb{R}^{d} \times \mathbb{S} ; \mathbb{R}_{+}\right)$, the family of all nonnegative functions $\mathbb{Q}$ is defined on $\mathbb{R}^{d} \times \mathbb{S}$ such that they are continuously twice differentiable in $\vartheta$. The operator $\mathscr{L}$ associated with $\left(^{*}\right)$ is defined as follows:

$$
\begin{aligned}
\mathscr{L} Q(\vartheta, i)= & \mathcal{Q}_{t}(t, \vartheta, i)+\mathcal{Q}_{\vartheta}(t, \vartheta, i) f(t, \vartheta, i) \\
& +\frac{1}{2} g^{T}(t, \vartheta, i) \mathscr{Q}_{\vartheta, \vartheta}(t, \vartheta, i) g(t, \vartheta, i) \\
& +\sum_{j \in \mathbb{S}} \phi_{i j} \mathcal{Q}(t, \vartheta, j),
\end{aligned}
$$

where $\mathbb{Q}_{\vartheta}$ and $\mathbb{Q}_{\vartheta, \vartheta}$ represent the gradient and Hessian of $\mathbb{Q}$ and $T$ is the transpose of a matrix.

By Itô's formula, if $\mathbb{Q}$ defined on $\mathbb{R}^{d} \times \mathbb{S}$, we have

$$
\mathrm{d} Q(\vartheta(t), i)=\mathscr{L} Q(\vartheta(t), i) \mathrm{d} t+\mathscr{Q}_{\vartheta}(\vartheta(t), i) g(\vartheta(t), i) \mathrm{d} B(t) .
$$

The total population in system (4) verifies the following equation:

$$
\begin{aligned}
\mathrm{d}(S(t) & \left.+I_{1}(t)+I_{2}(t)+R(t)\right) \\
& =\left[\mu-\mu\left(S(t)+I_{1}(t)+I_{2}(t)+R(t)\right)\right] \mathrm{d} t
\end{aligned}
$$

Thus,

$$
\begin{aligned}
S(t) & +I_{1}(t)+I_{2}(t)+R(t) \\
& =1+\left(S(0)+I_{1}(0)+I_{2}(0)+R(0)-1\right) e^{-\mu(r(t)) t}, \quad \text { for all } t \geq 0 .
\end{aligned}
$$

Since, for $X(0) \in \mathbb{D}$, we have

$$
S(t)+I_{1}(t)+I_{2}(t)+R(t)=1, \quad \text { for all } t \geq 0 \text { a.s. }
$$

Let

$$
\mathbb{D}=\left\{\left(S+I_{1}+I_{2}+R\right) \in \mathbb{R}_{+}^{4}: S+I_{1}+I_{2}+R=1\right\} .
$$

By using the same method as in [31, 32], we can easily prove the following theorem.

Theorem 1. Let $X(0)=\left(S(0), I_{1}(0), I_{2}(0), R(0)\right) \in \mathbb{D}$, then there is a unique solution $\left(S(t), I_{1}(t), I_{2}(t), R(t)\right)$ to system (4) on $t \geq 0$, and the solution will remain in $\mathbb{D}$ with probability 1 .

Firstly, we define the following expressions which we use to determine the extinction of epidemics: 


$$
\begin{aligned}
\sum_{i=1}^{m} \pi_{i} D_{i} & =\sum_{i=1}^{m} \pi_{i}\left(-\sigma_{1}^{2}(i)+\beta_{1}(i)-\left(\mu(i)+\gamma_{1}(i)\right)\right), \\
\sum_{i=1}^{m} \pi_{i} A_{i} & =\sum_{i=1}^{m} \pi_{i}\left(-\sigma_{2}^{2}(i)+\beta_{2}(i)-\left(\mu(i)+\gamma_{2}(i)\right)\right) .
\end{aligned}
$$

\section{Extinction}

This section is consecrated to give sufficient conditions so that the epidemics die out. According to [33], we have the following lemma.

Lemma 1. Suppose that there exists a function $U(u, i) \in C^{1,2}\left(\mathbb{R}^{3} \times \mathbb{S} ; \mathbb{R}_{+}\right)$and $\rho(u) \in\left(\mathbb{R}^{3} \times ; \mathbb{R}_{+}\right)$disappears only at $E_{0}$ such that

$$
\begin{array}{r}
\mathscr{L} U(u, i) \leq-\rho(u), \quad \text { for all }(u, i) \in \mathbb{R}^{3} \times \mathbb{S}, \\
\lim _{|x| \longrightarrow \infty} \inf _{j \in \mathcal{S}} U(u, i)=\infty .
\end{array}
$$

Then, the equilibrium $E_{0}$ of system (4) is globally asymptotically stable in probability.
By Lemma 1, we identify the conditions for stochastic asymptotic stability of the disease-free equilibrium which are presented by the following theorem.

Theorem 2. If $\beta_{2}(i) \geq \sigma_{2}^{2}(i)$ and $\beta_{1}(i) \geq \sigma_{1}^{2}(i)$ for all $i \in \mathbb{S}$, and

$$
\begin{aligned}
& \sum_{i=1}^{m} \pi_{i} D_{i}<0, \\
& \sum_{i=1}^{m} \pi_{i} A_{i}<0,
\end{aligned}
$$

then the disease-free equilibrium $E_{0}$ is globally stochastically asymptotically stable on $\mathbb{D}$.

Proof. We consider the following Lyapunov function:

$$
\begin{aligned}
\mathscr{H}(S, I, R, i)= & m_{1}(1-S)^{2}+\left(\eta+\omega_{i}\right) I_{1}^{(1 / \eta)} \\
& +\left(\theta+\Phi_{i}\right) I_{2}^{(1 / \theta)}+m_{2} R^{2}, \quad \text { for all } i \in \mathbb{S},
\end{aligned}
$$

where $m_{1}, m_{2}, \eta$, and $\theta$ are real positive constants to be determined later. By Itô's formula, we calculate $\mathscr{L} \mathscr{H}$ as follows:

$$
\begin{aligned}
& \mathscr{L} \mathscr{H}=-2 m_{1}(1-S)\left[\mu-\mu S-\beta_{1} S I_{1}-\beta_{2} S I_{2}+\delta R\right]+m_{1} \sigma_{1}^{2} S^{2} I_{1}^{2} \\
& +m_{2} \sigma_{2}^{2} S^{2} I_{2}^{2}+\frac{1}{\eta}\left(\eta+\omega_{i}\right) I_{1}^{(1 / \eta)}\left[\beta_{1} S-\left(\mu+\gamma_{1}\right)\right] \\
& +\frac{1}{2 \eta}\left(\frac{1}{\eta}-1\right)\left(\eta+\omega_{i}\right) \sigma_{1}^{2} S^{2} I_{1}^{(1 / \eta)} \\
& +\frac{1}{\theta}\left(\theta+{\omega_{i}}_{i} I_{2}^{(1 / \theta)}\left[\beta_{2} S-\left(\mu+\gamma_{2}\right)\right]+\frac{1}{2 \theta}\left(\frac{1}{\theta}-1\right)\left(\theta+\omega_{i}\right) \sigma_{2}^{2} S^{2} I_{2}^{(1 / \theta)}\right. \\
& +2 m_{2} R\left[\gamma_{1} I_{1}+\gamma_{2} I_{2}-(\mu+\delta) R\right]+\sum_{k \neq i, k \in \mathbb{S}} \phi_{i k}\left(\omega_{k}-\omega_{i}+\varpi_{k}-\varpi_{i}\right) \\
& =-2 m_{1}(1-S)^{2}+2 m_{1} \beta_{1} S I_{1}(1-S)+2 m_{1} \beta_{2} S I_{2}(1-S) \\
& -2 m_{1} \delta(1-S) R+m_{1} \sigma_{1} S^{2} I_{1}^{2}+m_{1} \sigma_{2} S^{2} I_{2}^{2}+\frac{1}{\eta}\left(\eta+\omega_{i}\right) \beta_{1} I_{1}^{(1 / \eta)} S \\
& -\frac{1}{\eta}\left(\eta+\omega_{i}\right)\left(\mu+\gamma_{1}\right) I_{1}^{(1 / \eta)}+\frac{1}{2 \eta}\left(\frac{1}{\eta}-1\right)\left(\eta+\omega_{i}\right) \sigma_{1}^{2} S^{2} I_{1}^{(1 / \eta)} \\
& +\frac{1}{\theta}\left(\theta+\varpi_{i}\right) \beta_{2} I_{2}^{(1 / \theta)} S-\frac{1}{\theta}\left(\theta+\varpi_{i}\right)\left(\mu+\gamma_{2}\right) I_{2}^{(1 / \theta)} \\
& +\frac{1}{2 \theta}\left(\frac{1}{\theta}-1\right)\left(\theta+\omega_{i}\right) \sigma_{2}^{2} S^{2} I_{2}^{(1 / \theta)}+m_{2} \gamma_{1} R I_{1}+m_{2} \gamma_{2} R I_{2}-m_{2}(\mu+\delta) R^{2} \\
& +I_{1}^{(1 / \eta)} \sum_{k \neq i, k \in \mathbb{S}} \phi_{i k}\left(\omega_{k}-\omega_{i}\right)+I_{2}^{(1 / \theta)} \sum_{k \neq i, k \in \mathbb{S}} \phi_{i k}\left(\varpi_{k}-\varpi_{i}\right) .
\end{aligned}
$$


Using the fact that $I \leq 1-S$ and $S, I \in \mathbb{D}$, we obtain

$$
\begin{aligned}
\mathscr{L} \mathscr{H} \leq & -2 m_{1}(1-S)^{2}+2 m_{1} \beta_{1} I_{1}^{(1 / \eta)}+2 m_{1} \beta_{2} I_{2}^{(1 / \theta)}+m_{1} \sigma_{1}^{2} I_{1}^{(1 / \eta)}+m_{1} \sigma_{2}^{2} I_{2}^{(1 / \theta)} \\
& +\frac{1}{\eta}\left(\eta+\omega_{i}\right) \beta_{1} I_{1}^{(1 / \eta)} S-\frac{1}{\eta}\left(\eta+\omega_{i}\right)\left(\mu+\gamma_{1}\right) I_{1}^{(1 / \eta)} \\
& +\frac{1}{2 \eta}\left(\frac{1}{\eta}-1\right)\left(\eta+\omega_{i}\right) \sigma_{1}^{2} S^{2} I^{(1 / \eta)}+\frac{1}{\theta}\left(\theta+\varpi_{i}\right) \beta_{2} I_{2}^{(1 / \theta)} S \\
& -\frac{1}{\theta}\left(\theta+\varpi_{i}\right)\left(\mu+\gamma_{2}\right) I_{2}^{(1 / \theta)}+\frac{1}{2 \theta}\left(\frac{1}{\theta}-1\right)\left(\theta+\omega_{i}\right) \sigma_{2}^{2} S^{2} I_{2}^{(1 / \theta)} \\
& +\left(m_{2} \gamma_{1}-m_{1} \delta\right) R I_{1}+\left(m_{2} \gamma_{2}-m_{1} \delta\right) R I_{2}-m_{2}(\mu+\delta) R^{2} \\
& +I_{1}^{(1 / \eta)} \sum_{k \neq i, k \in \mathbb{S}} \phi_{i k}\left(\omega_{k}-\omega_{i}\right)+I_{2}^{(1 / \theta)} \sum_{k \neq i, k \in \mathbb{S}} \phi_{i k}\left(\varpi_{k}-\oplus_{i}\right),
\end{aligned}
$$

for all $\eta$ and $\theta \geq 1$. Since we can choose $m_{2}<\min _{i \in \mathbb{S}}\left\{\left(m_{1} \delta(i) / \gamma_{1}(i)\right),\left(m_{1} \delta(i) / \gamma_{2}(i)\right)\right\}$, we obtain

$$
\begin{aligned}
& \mathscr{L} \mathscr{H} \leq-2 m_{1}(1-S)^{2}-m_{2}(\mu+\delta) R^{2}+\frac{\left(\eta+\omega_{i}\right)}{\eta} I_{1}^{(1 / \eta)}\left[\frac{\eta m_{1}}{\left(\eta+\omega_{i}\right)}\left(\sigma_{1}^{2}+2 \beta_{1}\right)\right. \\
& \left.-\left(\mu+\gamma_{1}\right)+\frac{1}{2 \eta} \sigma_{1}^{2}+\beta_{1} S-\sigma_{1}^{2} S^{2}+\frac{\eta}{\left(\eta+\omega_{i}\right.} \sum_{k \neq i, k \in \mathbb{S}} \phi_{i k}\left(\omega_{k}-\omega_{i}\right)\right] \\
& +\frac{\left(\theta+\omega_{i}\right)}{\theta} I_{2}^{(1 / \theta)}\left[\frac{\theta m_{1}}{\left(\theta+\omega_{i}\right)}\left(\sigma_{2}^{2}+2 \beta_{2}\right)-\left(\mu+\gamma_{2}\right)+\frac{1}{2 \theta} \sigma_{2}^{2}+\beta_{2} S\right. \\
& \left.-\sigma_{2}^{2} S^{2}+\frac{\theta}{\left(\theta+\varpi_{i}\right)} \sum_{k \neq i, k \in \mathbb{S}} \phi_{i k}\left(\varpi_{k}-\varpi_{i}\right)\right] \\
& =:-2 m_{1}(1-S)^{2}-m_{2}(\mu+\delta) R^{2}+\frac{\left(\eta+\omega_{i}\right)}{\eta} I_{1}^{(1 / \eta)}\left[\frac{\eta m_{1}}{\left(\eta+\omega_{i}\right)}\left(\sigma_{1}^{2}+2 \beta_{1}\right)\right. \\
& \left.+\frac{1}{2 \eta} \sigma_{1}^{2}+f_{i}(S)+\frac{\eta}{\left(\eta+\omega_{i}\right)} \sum_{k \neq i, k \in \mathbb{S}} \phi_{i k}\left(\omega_{k}-\omega_{i}\right)\right] \\
& +\frac{\left(\theta+\omega_{i}\right)}{\theta} I_{2}^{(1 / \theta)}\left[\frac{\theta m_{1}}{\left(\theta+\omega_{i}\right)}\left(\sigma_{2}^{2}+2 \beta_{2}\right)+\frac{1}{2 \theta} \sigma_{2}^{2}+g_{i}(S)\right. \\
& \left.+\frac{\theta}{\left(\theta+\varpi_{i}\right)} \sum_{k \neq i, k \in \mathbb{S}} \phi_{i k}\left(\varpi_{k}-\varpi_{i}\right)\right] \text {, }
\end{aligned}
$$

where $\quad f_{i}(x)=-\sigma_{1}^{2}(i) x^{2}+\beta_{1}(i) x-\left(\mu(i)+\gamma_{1}(i)\right) \quad$ and $g_{i}(x)=-\sigma_{2}^{2}(i) x^{2}+\beta_{2}(i) x-\left(\mu(i)+\gamma_{2}(i)\right)$. Note that the functions $f_{i}(x)$ and $g_{i}(x)$ are all increasing on $(0,1)$, which means

$$
\begin{aligned}
& f_{i}(x) \leq f_{i}(1)=: D_{i}, \\
& g_{i}(x) \leq g_{i}(1)=: A_{i},
\end{aligned}
$$

then 


$$
\begin{aligned}
\mathscr{L} \mathscr{H} \leq & -2 m_{1}(1-S)^{2}-m_{2}(\mu+\delta) R^{2}+\frac{\left(\eta+\omega_{i}\right)}{\eta} I_{1}^{(1 / \eta)}\left[\frac{\eta m_{1}}{\left(\eta+\omega_{i}\right)}\left(\sigma_{1}^{2}+2 \beta_{1}\right)\right. \\
& \left.+\frac{1}{2 \eta} \sigma_{1}^{2}+D_{i}+\frac{\eta}{\left(\eta+\omega_{i}\right)} \sum_{k \neq i, k \in \mathbb{S}} \phi_{i k}\left(\omega_{k}-\omega_{i}\right)\right] \\
& +\frac{\left(\theta+\Phi_{i}\right)}{\theta} I_{2}^{(1 / \theta)}\left[\frac{\theta m_{1}}{\left(\theta+\varpi_{i}\right)}\left(\sigma_{2}^{2}+2 \beta_{2}\right)+\frac{1}{2 \theta} \sigma_{2}^{2}+A_{i}\right. \\
& \left.+\frac{\theta}{\left(\theta+\Phi_{i}\right)} \sum_{k \neq i, k \in \mathbb{S}} \phi_{i k}\left(\varpi_{k}-\Phi_{i}\right)\right] .
\end{aligned}
$$

Since the generator matrix $\Phi$ is irreducible, then for $D=\left(D_{1}, \ldots, D_{m}\right)^{T}$, there exists $\Xi=\left(\omega_{1}, \ldots, \omega_{m}\right)^{T}$ solution of the Poisson system:

$$
\Phi \Xi=-D+\left(\sum_{i=1}^{m} \pi_{i} D_{i}\right) \vec{E}
$$

And for $A=\left(A_{1}, \ldots, A_{m}\right)^{T}$, there exists $\Theta=\left(\varpi_{1}, \ldots, \varpi_{m}\right)^{T}$ solution of the Poisson system:

$$
\Phi \Theta=-A+\left(\sum_{i=1}^{m} \pi_{i} A_{i}\right) \vec{E},
$$

where $\vec{E}$ denotes the column vector with all its entries equal to 1 .

Substituting (23) and (24) in (22), we get

$$
\begin{aligned}
\mathscr{L} \mathscr{H} \leq & -2 m_{1}(1-S)^{2}-m_{2}(\mu+\delta) R^{2}+\frac{\left(\eta+\omega_{i}\right)}{\eta} I_{1}^{(1 / \eta)}\left[\frac{\eta m_{1}}{\left(\eta+\omega_{i}\right)}\left(\sigma_{1}^{2}+2 \beta_{1}\right)\right. \\
& \left.+\frac{1}{2 \eta} \sigma_{1}^{2}+\frac{\eta}{\left(\eta+\omega_{i}\right)}\left(D_{i}-\sum_{i=1}^{m} \pi_{i} D_{i}\right)+\sum_{i=1}^{m} \pi_{i} D_{i}\right] \\
& +\frac{\left(\theta+\omega_{i}\right)}{\theta} I_{2}^{(1 / \theta)}\left[\frac{\theta m_{1}}{\left(\theta+\Phi_{i}\right)}\left(\sigma_{2}^{2}+2 \beta_{2}\right)+\frac{1}{2 \theta} \sigma_{2}^{2}\right. \\
& \left.+\frac{\theta}{\left(\theta+\Phi_{i}\right)}\left(A_{i}-\sum_{i=1}^{m} \pi_{i} A_{i}\right)+\sum_{i=1}^{m} \pi_{i} A_{i}\right] .
\end{aligned}
$$

Choosing $\eta_{0}$ and $\theta_{0}$, such that

$$
\begin{gathered}
C_{1}=: \max _{i \in \mathbb{S}}\left\{\frac{\sigma_{1}^{2}}{2 \eta_{0}}+\frac{\eta_{0}}{\left(\eta_{0}+\omega_{i}\right)}\left(D_{i}-\sum_{i=1}^{m} \pi_{i} D_{i}\right)+\sum_{i=1}^{m} \pi_{i} D_{i}\right\}<0, \\
C_{2}:=\max _{i \in \mathbb{S}}\left\{\frac{\sigma_{2}^{2}}{2 \theta_{0}}+\frac{\theta_{0}}{\left(\theta_{0}+\Phi_{i}\right)}\left(A_{i}-\sum_{i=1}^{m} \pi_{i} A_{i}\right)+\sum_{i=1}^{m} \pi_{i} A_{i}\right\}<0 .
\end{gathered}
$$

Since we choose $\eta>\max \left\{-\omega_{i}, \eta_{0}, 1\right\} \quad$ and $\theta>\max \left\{-\omega_{i}, \theta_{0}, 1\right\}$ such that

$$
\begin{gathered}
m_{1}<\min \left\{-\frac{\eta+\min \omega_{i}}{\eta\left(\max \sigma_{1}^{2}+2 \max \beta_{1}\right)} \times C_{1},\right. \\
\left.-\frac{\theta+\min \omega_{i}}{\theta\left(\max \sigma_{2}^{2}+2 \max \beta_{2}\right)} \times C_{2}\right\} .
\end{gathered}
$$

According to Lemma 1, the proof is completed.

Theorem 3. Let $\left(S(t), I_{1}(t), I_{2}(t), R(t)\right)$ be the solution of system (4), then for any given initial value $\left(S(0), I_{1}\right.$ $\left.(0), I_{2}(0), R(0)\right) \in \mathbb{D}$, model (4) has the following property:

$$
\lim _{t \rightarrow \longrightarrow \infty} \frac{1}{t} \ln \left(1-S+I_{1}+I_{2}+R\right)
$$

$$
\leq \sum_{i=1}^{m} \pi_{i}\left(\frac{\beta_{1}^{2}(i)-\mu(i) \sigma_{1}^{2}(i)}{2 \sigma_{1}^{2}(i)}+\frac{\beta_{2}^{2}(i)-\mu(i) \sigma_{2}^{2}(i)}{2 \sigma_{2}^{2}(i)}\right), \quad \text { a.s. }
$$

$$
\begin{aligned}
& \text { If } \\
& \sum_{i=1}^{m} \pi_{i}\left(\frac{\beta_{1}^{2}(i)-\mu(i) \sigma_{1}^{2}(i)}{2 \sigma_{1}^{2}(i)}+\frac{\beta_{2}^{2}(i)-\mu(i) \sigma_{2}^{2}(i)}{2 \sigma_{2}^{2}(i)}\right)<0
\end{aligned}
$$


holds, then the disease-free $E_{0}$ is almost surely exponentially stable in $\mathbb{D}$. Namely, the diseases die out with probability one.

Proof. Consider the following function:

$$
\mathscr{G}\left(S, I_{1}, I_{2}, R, i\right)=\ln \left(1-S+I_{1}+I_{2}+R\right)
$$

By Itô's formula, we obtain

$$
\begin{aligned}
\mathrm{d} \mathscr{G} & =\frac{1}{1-S+I_{1}+I_{2}+R}\left[-\mu(r)(1-S)-\mu(r) I_{1}-\mu(r) I_{2}\right. \\
& \left.-(\mu(r)+2 \delta(r))(r) R+2 \beta_{1}(r) S I_{1}+2 \beta_{2}(r) S I_{2}\right] \mathrm{d} t \\
& -2 \sigma_{1}^{2}(r)\left(\frac{S I_{1}}{1-S+I_{1}+I_{2}+R}\right)^{2} \mathrm{~d} t-2 \sigma_{2}^{2}(r)\left(\frac{S I_{2}}{1-S+I_{1}+I_{2}+R}\right)^{2} \mathrm{~d} t \\
& +\frac{2 \sigma_{1}(r) S I_{1}}{1-S+I_{1}+I_{2}+R} \mathrm{~d} B_{1}+\frac{2 \sigma_{2}(r) S I_{2}}{1-S+I_{1}+I_{2}+R} \mathrm{~d} B_{2} .
\end{aligned}
$$

Note that

$$
\frac{1}{\left(1-S+I_{1}+I_{2}+R\right)}\left[-\mu(r)(1-S)-\mu(r) I_{1}-\mu I_{2}-(\mu(r)+2 \delta(r)) R\right] \leq-\mu(r) .
$$

Thus, we have

$$
\begin{aligned}
d \mathscr{G} \leq & \left(-2 \sigma_{1}(r)\left(\frac{S I}{1-S+I_{1}+I_{2}+R}-\frac{\beta_{1}(r)}{2 \sigma_{1}^{2}(r)}\right)+\frac{\beta_{1}^{2}(r)}{2 \sigma_{1}^{2}(r)}-\frac{\mu(r)}{2}\right) d t \\
& +\left(-2 \sigma_{2}(r)\left(\frac{S I}{1-S+I_{1}+I_{2}+R}-\frac{\beta_{2}(r)}{2 \sigma_{2}^{2}(r)}\right)+\frac{\beta_{2}^{2}(r)}{2 \sigma_{2}^{2}(r)}-\frac{\mu(r)}{2}\right) d t \\
& +\frac{2 \sigma_{1}(r) S I_{1}}{1-S+I_{1}+I_{2}+R} d B_{1}+\frac{2 \sigma_{2}(r) S I_{2}}{1-S+I_{1}+I_{2}+R} d B_{2} . \\
\leq & {\left[\frac{\beta_{1}^{2}(r)-\mu(r) \sigma_{1}^{2}(r)}{2 \sigma_{1}^{2}(r)}+\frac{\beta_{2}^{2}(r)-\mu(r) \sigma_{2}^{2}(r)}{2 \sigma_{2}^{2}(r)}\right] d t+\frac{2 \sigma_{1}(r) S I_{1}}{1-S+I_{1}+I_{2}+R} d B_{1} } \\
& +\frac{2 \sigma_{2}(r) S I_{2}}{1-S+I_{1}+I_{2}+R} d B_{2} .
\end{aligned}
$$


Integrating both sides of (33) from 0 to $t$ and dividing by t, we get

$$
\begin{aligned}
\frac{\mathscr{G}(t)}{t} \leq & \frac{\mathscr{G}(0)}{t} \\
& +\frac{1}{t} \int_{0}^{t}\left(\frac{\beta_{1}^{2}(r(u))-\mu(r(u)) \sigma_{1}^{2}(r(u))}{2 \sigma_{1}^{2}(r(u))}+\frac{\beta_{2}^{2}(r(u))-\mu(r(u)) \sigma_{2}^{2}(r(u))}{2 \sigma_{2}^{2}(r(u))}\right) \mathrm{d} u \\
& +\frac{1}{t} \int_{0}^{t} \frac{2 \sigma_{1}(r(u)) S(u) I_{1}(u)}{1-S(u)+I_{1}(u)+I_{2}(u)+R(u)} \mathrm{d} B_{1}(u) \\
& +\frac{1}{t} \int_{0}^{t} \frac{2 \sigma_{2}(r(u)) S(u) I_{2}(u)}{1-S(u)+I_{1}(u)+I_{2}(u)+R(u)} \mathrm{d} B_{2}(u)
\end{aligned}
$$

Let $\quad F_{i}(t)=\int_{0}^{t}\left(\left(2 \sigma_{i}(r) S(u) I_{i}(u)\right) /\left(1-S(u)+I_{1}(u)+\right.\right.$ $\left.\left.I_{2}(u)+R(u)\right)\right) \mathrm{d} B_{i}(u)(i=1,2)$, then

$$
\begin{aligned}
& \left\langle F_{i}(t), F_{i}(t)\right\rangle \\
& =\int_{0}^{t}\left(\frac{2 \sigma_{i}(r(u)) S(u) I_{i}(u)}{1-S(u)+I_{1}(u)+I_{2}(u)+R(u)}\right)^{2} \mathrm{~d} u \leq 4 \check{\sigma}_{i}, \quad t<\infty .
\end{aligned}
$$

Then, by strong law of large numbers, we obtain

$$
\lim _{t \rightarrow \infty} \frac{F_{i}(t)}{t}=0, \quad \text { a.s. }
$$

By the ergodic theory of the Markov chain, we can obtain

$$
\begin{aligned}
& \limsup _{t \longrightarrow \infty} \frac{1}{t} \int_{0}^{t}\left(\frac{\beta_{1}^{2}(r(u))-\mu(r(u)) \sigma_{1}^{2}(r(u))}{2 \sigma_{1}^{2}(r(u))}+\frac{\beta_{2}^{2}(r(u))-\mu(r(u)) \sigma_{2}^{2}(r(u))}{2 \sigma_{2}^{2}(r(u))}\right) \mathrm{d} u \\
& \leq \sum_{i=1}^{m} \pi_{i}\left(\frac{\beta_{1}^{2}(i)-\mu(i) \sigma_{1}^{2}(i)}{2 \sigma_{1}^{2}(i)}+\frac{\beta_{2}^{2}(i)-\mu(i) \sigma_{2}^{2}(i)}{2 \sigma_{2}^{2}(i)}\right), \quad \text { a.s. }
\end{aligned}
$$

Hence, if $\sum_{i=1}^{m} \pi_{i}\left(\left(\left(\beta_{1}^{2}(i)-\mu(i) \sigma_{1}^{2}(i)\right) / 2 \sigma_{1}^{2}(i)\right)+\left(\left(\beta_{2}^{2}(i)-\right.\right.\right.$ $\left.\left.\left.\mu(i) \sigma_{2}^{2}(i)\right) / 2 \sigma_{2}^{2}(i)\right)\right)<0$, then the disease-free $E_{0}$ is almost surely exponentially stable in $\mathbb{D}$.

\section{Persistence}

In this section, we explain sufficient conditions for the persistence of two epidemics.

We define $\langle h\rangle_{t}=(1 / t) \int_{0}^{t} h(u) \mathrm{d} u$ if $h$ is an integrable function on $[0,+\infty)$.

Definition 1. System (4) is said to be persistent in the mean, if

$$
\liminf _{t \rightarrow \infty} \frac{1}{t} \int_{0}^{t} I(u) \mathrm{d} u>0, \quad \text { a.s. }
$$

Let

$$
R_{0}^{s}=\sum_{i=0}^{m} \pi_{i}\left(2 \mu(i)+\gamma_{1}(i)+\gamma_{2}(i)+\frac{\sigma_{1}(i)}{2}+\frac{\sigma_{2}(i)}{2}-k_{1}(i) \mu(i)\right) \text {. }
$$

Theorem 4. Let $\left(S(t), I_{1}(t), I_{2}(t), R(t)\right)$ be the solution of model (4). If $R_{0}^{s}>0$, then

$$
\begin{aligned}
\liminf _{t \rightarrow \infty}\left\langle I_{1}+I_{2}\right\rangle_{t} & \geq I^{*}, \\
\liminf _{t \rightarrow \infty}\langle R\rangle_{t} & \geq \frac{\min \left\{\widehat{\gamma}_{1}, \widehat{\gamma}_{2}\right\}}{\check{\mu}+\check{\delta}} I^{*}, \\
\liminf _{t \rightarrow \infty}\langle 1-S\rangle_{t} & \geq\left(\frac{\check{\mu}+\check{\delta}+\min \left\{\widehat{\gamma}_{1}, \widehat{\gamma}_{2}\right\}}{\check{\mu}+\check{\delta}}\right) I^{*},
\end{aligned}
$$

where $I^{*}=\left(R_{0}^{s} / \bar{D}\right)$ with $\bar{D}$ is positive constant.

Proof. By system (4), one can obtain that

$$
\begin{aligned}
& \mathscr{L}\left(-\ln I_{1}\right)=-\beta_{1}(i) S+\mu(i)+\gamma_{1}(i)+\frac{\sigma_{1}^{2}(i)}{2} S^{2}, \\
& \mathscr{L}\left(-\ln I_{2}\right)=-\beta_{2}(i) S+\mu(i)+\gamma_{2}(i)+\frac{\sigma_{2}^{2}(i)}{2} S^{2} .
\end{aligned}
$$




$$
\begin{aligned}
\mathscr{L}\left(S+I_{1}+I_{2}\right)= & \mu(i)-\mu(i) S-\left(\mu(i)+\gamma_{1}(i)\right) \\
& I_{1}-\left(\mu(i)+\gamma_{2}(i)\right) I_{2}+\delta(i) R, \\
\mathscr{L} R= & \gamma_{1}(i) I_{1}+\gamma_{2}(i) I_{2}-(\mu(i)+\delta(i)) R .
\end{aligned}
$$
by

So, we consider the Lyapunov function $\mathscr{T}$ defined on $\mathbb{D}$

$$
\begin{aligned}
\mathscr{T}\left(S, I_{1}, I_{2}, i\right)= & -\ln I_{1}-\ln I_{2} \\
& -k_{1}(i)\left(S+I_{1}+I_{2}\right)-k_{2}(i) R-v(i),
\end{aligned}
$$

$$
\begin{array}{r}
\beta_{1}(i)+\beta_{2}(i)-k_{1}(i) \mu(i)+\sum_{l=1}^{m} \phi_{i l} k_{1}(l)=0, \\
k_{1}(i) \delta(i)-k_{2}(i)(\mu(i)+\delta(i))+\sum_{l=1}^{m} \phi_{i l} k_{2}(l)=0 .
\end{array}
$$

Using Itô's formula, one can get that

$$
\begin{aligned}
\mathrm{d} \mathscr{T}= & \mathscr{L} \mathscr{T} \mathrm{d} t-\left[\sigma_{1}(i) S+k_{1}(i) S I_{1}\right] \mathrm{d} B_{1}(t) \\
& -\left[\sigma_{2}(i) S+k_{2}(i) S I_{2}\right] \mathrm{d} B_{2}(t),
\end{aligned}
$$

where

where $\left(k_{1}(i), k_{2}(i)\right)^{T}$ is the unique positive solution of the following system:

$$
\begin{aligned}
\mathscr{L} \mathscr{T}= & -\beta_{1} S+\mu(i)+\gamma_{1}(i)+\frac{\sigma_{1}^{2}(i)}{2} S^{2}-\beta_{2}(i) S+\mu(i)+\gamma_{2}(i)+\frac{\sigma_{2}^{2}(i)}{2} S^{2} \\
& -k_{1}(i)\left[\mu(i)-\mu(i) S-\left(\mu(i)+\gamma_{1}(i)\right) I_{1}-\left(\mu(i)+\gamma_{2}(i)\right) I_{2}+\delta(i) R\right] \\
& -k_{2}(i)\left[\gamma_{1}(i) I_{1}+\gamma_{2}(i) I_{2}-(\mu(i)+\delta(i)) R\right]-\sum_{l=1}^{m} \phi_{i l} k_{1}(l)\left(S+I_{1}+I_{2}\right) \\
& -\sum_{l=1}^{m} \phi_{i l} k_{2}(l) R-\sum_{l=1}^{m} \phi_{i l} v(l) .
\end{aligned}
$$

Using the fact that $S \in(0,1)$, we deduce that

$$
\begin{aligned}
\mathscr{L} \mathscr{T} \leq & 2 \mu(i)+\gamma_{1}(i)+\gamma_{2}(i)+\frac{\sigma_{1}^{2}(i)}{2}+\frac{\sigma_{2}^{2}(i)}{2}-k_{1}(i) \mu(i)-\sum_{l=1}^{m} \phi_{i l} v(l) \\
& -\left[\beta_{1}(i)+\beta_{2}(i)-k_{1}(i) \mu(i)+\sum_{l=1}^{m} \phi_{i l} k_{1}(l)\right] S \\
& -\left[k_{1}(i) \delta(i)-k_{2}(i)(\mu(i)+\delta(i))+\sum_{l=1}^{m} \phi_{i l} k_{2}(l)\right] R \\
& +\left[k_{1}(i)\left(\mu(i)+\gamma_{1}(i)\right)-k_{2}(i) \gamma_{1}(i)-\sum_{l=1}^{m} \phi_{i l} k_{1}(l)\right] I_{1} \\
& +\left[k_{1}(i)\left(\mu(i)+\gamma_{2}(i)\right)-k_{2}(i) \gamma_{2}(i)+\sum_{l=1}^{m} \phi_{i l} k_{1}(l)\right] I_{2} .
\end{aligned}
$$


From (44), we get that

$$
\begin{aligned}
\mathscr{L} \mathscr{T} \leq & 2 \mu(i)+\gamma_{1}(i)+\gamma_{2}(i)+\frac{\sigma_{1}^{2}(i)}{2}+\frac{\sigma_{2}^{2}(i)}{2}-k_{1}(i) \mu(i)-\sum_{l=1}^{m} \phi_{i l} v(l) \\
& +\left[\beta_{1}(i)+\beta_{2}(i)+k_{1}(i) \gamma_{1}(i)\right] I_{1}+\left[\beta_{1}(i)+\beta_{2}(i)+k_{1}(i) \gamma_{2}(i)\right] I_{2} .
\end{aligned}
$$

In addition, we also have

$$
\begin{aligned}
\mathscr{L} \mathscr{T} \leq & \mu(i)+\gamma_{1}(i)+\gamma_{2}(i)+\frac{\sigma_{1}^{2}(i)}{2}+\frac{\sigma_{2}^{2}(i)}{2}-k_{1}(i) \mu(i)-\sum_{l=1}^{m} \phi_{i l} v(l) \\
& +\left[\check{\beta}_{1}+\check{\beta}_{2}+\check{k}_{1} \check{\gamma}_{1}\right] I_{1}+\left[\check{\beta}_{1}+\check{\beta}_{2}+\check{k}_{1} \check{\gamma}_{2}\right] I_{2} \\
\leq & 2 \mu(i)+\gamma_{1}(i)+\gamma_{2}(i)+\frac{\sigma_{1}^{2}(i)}{2}+\frac{\sigma_{2}^{2}(i)}{2}-k_{1}(i) \mu(i)-\sum_{l=1}^{m} \phi_{i l} v(l) \phi_{i l} v(l)+\bar{D}\left(I_{1}+I_{2}\right), \\
& +\bar{D}\left(I_{1}+I_{2}\right) \\
:= & -R_{0}(i)-\sum_{l=1}^{m}
\end{aligned}
$$

where

$$
\bar{D}=\max \left\{\check{\beta}_{1}+\check{\beta}_{2}+\check{k}_{1} \check{\gamma}_{1}, \check{\beta}_{1}+\check{\beta}_{2}+\check{k}_{1} \check{\gamma}_{2}\right\} .
$$

Since the generator matrix $\Phi$ is irreducible, then for $R_{0}=\left(R_{0}(1), \ldots, R_{0}(m)\right)^{T}$, there exists $\Upsilon=(v(1)$, $\ldots, v(m))^{T}$ solution of the Poisson system:

$$
\Phi \Upsilon=-R_{0}+\left(\sum_{i=1}^{m} \pi_{i} R_{0}(i)\right)\left(\begin{array}{c}
1 \\
1 \\
\cdot \\
\cdot \\
1
\end{array}\right) \text {, }
$$

which implies that

$$
\mathscr{L} \mathscr{T} \leq-R_{0}^{s}+\bar{D}\left(I_{1}+I_{2}\right)
$$

Integrating (45) from 0 to $t$ and dividing by $t$ on both sides, we obtain

$$
\frac{\mathscr{T}(t)}{t} \leq \frac{\mathscr{T}(0)}{t}-R_{0}^{s}+\bar{D}\left\langle I_{1}+I_{2}\right\rangle_{t}-\frac{M_{1}(t)}{t}-\frac{M_{2}(t)}{t},
$$

where $M_{j}(t)=\sigma_{j}(i) S \mathrm{~d} B_{j}(t)$ (for $j=1,2$ ) is a continuous martingale with $M(0)=0$ and its quadratic variation is as follows:

$$
\left\langle M_{j}(t), M_{j}(t)\right\rangle=\int_{0}^{t}\left(\sigma_{j}(i) S(u)\right)^{2} \mathrm{~d} u \leq\left(\check{\sigma}_{j}\right)^{2} t .
$$

Thus, the strong law of large number for local martingales [27] implies that

$$
\lim _{t \rightarrow \infty} \frac{M_{j}(t)}{t}=0, \quad \text { a.s. }
$$

Since $\left(S, I_{1}, I_{1}, R\right) \in \mathbb{D}$, we can derive

$$
\begin{aligned}
\mathscr{T}\left(S, I_{1}, I_{2}, i\right)= & -\ln I_{1}-\ln I_{2}-k_{1}(i)\left(S+I_{1}+I_{2}\right) \\
& -k_{2}(i) R-v(i) \geq-\check{k}_{1}-\check{k}_{2}-\check{v}:=C .
\end{aligned}
$$

Taking the superior limit on both sides of (45) and combining with (52), (55), and (56), we deduce that

$$
\liminf _{t \longrightarrow \infty}\left\langle I_{1}+I_{2}\right\rangle_{t} \geq \frac{R_{0}^{s}}{\bar{D}}:=I^{*}, \quad \text { a.s. }
$$

Integrating the third equation of (4) and dividing both sides by $t$, we have

$$
\langle R\rangle_{t} \geq \frac{\min \left\{\widehat{\gamma}_{1}, \widehat{\gamma}_{2}\right\}}{(\check{\mu}+\check{\delta})}\left\langle I_{1}+I_{2}\right\rangle_{t}-\frac{R(t)-R(0)}{(\widehat{\mu}+\widehat{\delta}) t} .
$$

Since $\lim _{t \rightarrow \infty}((R(t)-R(0)) / t)=0$ and using (57), we get

$$
\liminf _{t \rightarrow \infty}\langle R\rangle_{t} \geq \frac{\min \left\{\widehat{\gamma}_{1}, \widehat{\gamma}_{2}\right\}}{\check{\mu}+\check{\delta}} I^{*}
$$

Furthermore, it follows by $1-S=I+R$ that 
TABle 1: Parameter values used in numerical simulations.

\begin{tabular}{lcccccccc}
\hline States & Coefficients & & & & & & \\
& $\mu$ & $\beta_{1}$ & $\beta_{2}$ & $\delta$ & $\gamma_{1}$ & $\gamma_{2}$ & $\sigma_{1}$ & $\sigma_{2}$ \\
\hline 1 & 0.25 & 0.5 & 0.6 & 0.5 & 0.4 & 0.3 & 0.2 & 0.1 \\
2 & 0.3 & 0.6 & 0.4 & 0.4 & 0.2 & 0.4 & 0.12 & 0.2 \\
\hline
\end{tabular}
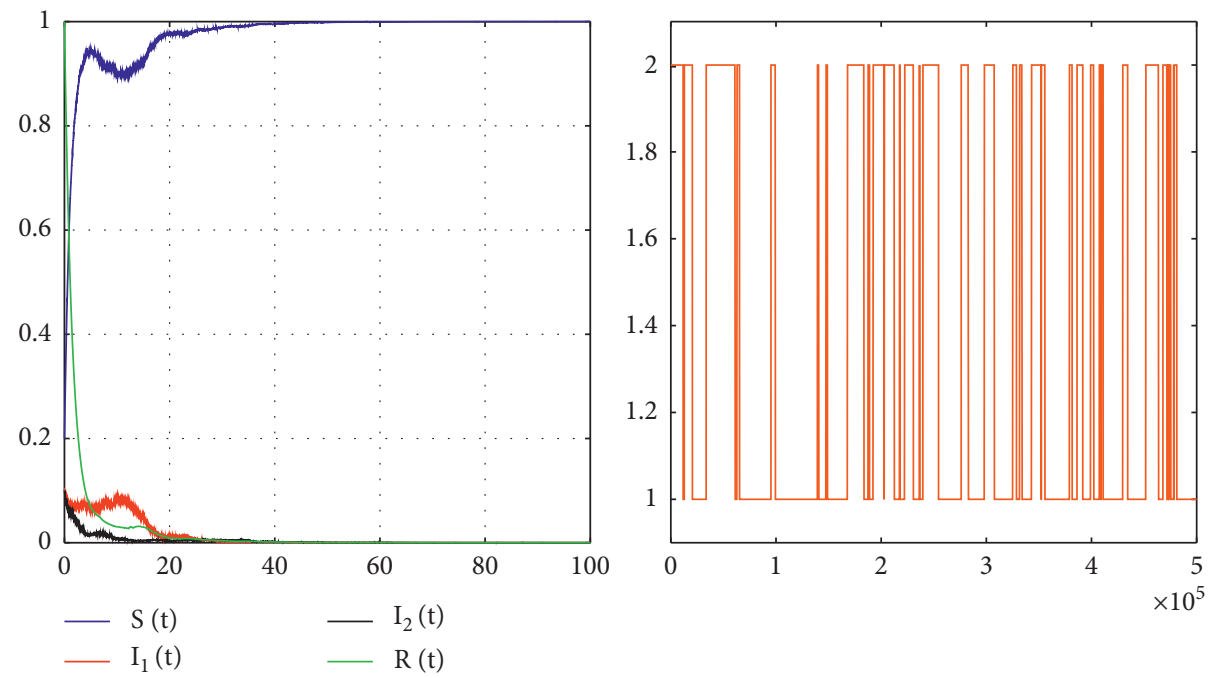

FIgURE 2: Sample paths of $S(t)$ in blue, $I_{1}(t)$ in red, $I_{2}(t)$ in black, $R(t)$ in green, and $r(t)$ in brown in Example 1.

TABLE 2: Parameter values used in numerical simulations.

\begin{tabular}{lcccccccc}
\hline States & Coefficients & & & & & & \\
& $\mu$ & $\beta_{1}$ & $\beta_{2}$ & $\delta$ & $\gamma_{1}$ & $\gamma_{2}$ & $\sigma_{1}$ & $\sigma_{2}$ \\
\hline 1 & 0.01 & 0.9 & 0.8 & 0.4 & 0.2 & 0.3 & 0.6 & 0.8 \\
2 & 0.01 & 0.8 & 0.8 & 0.4 & 0.3 & 0.2 & 0.7 & 0.7 \\
\hline
\end{tabular}
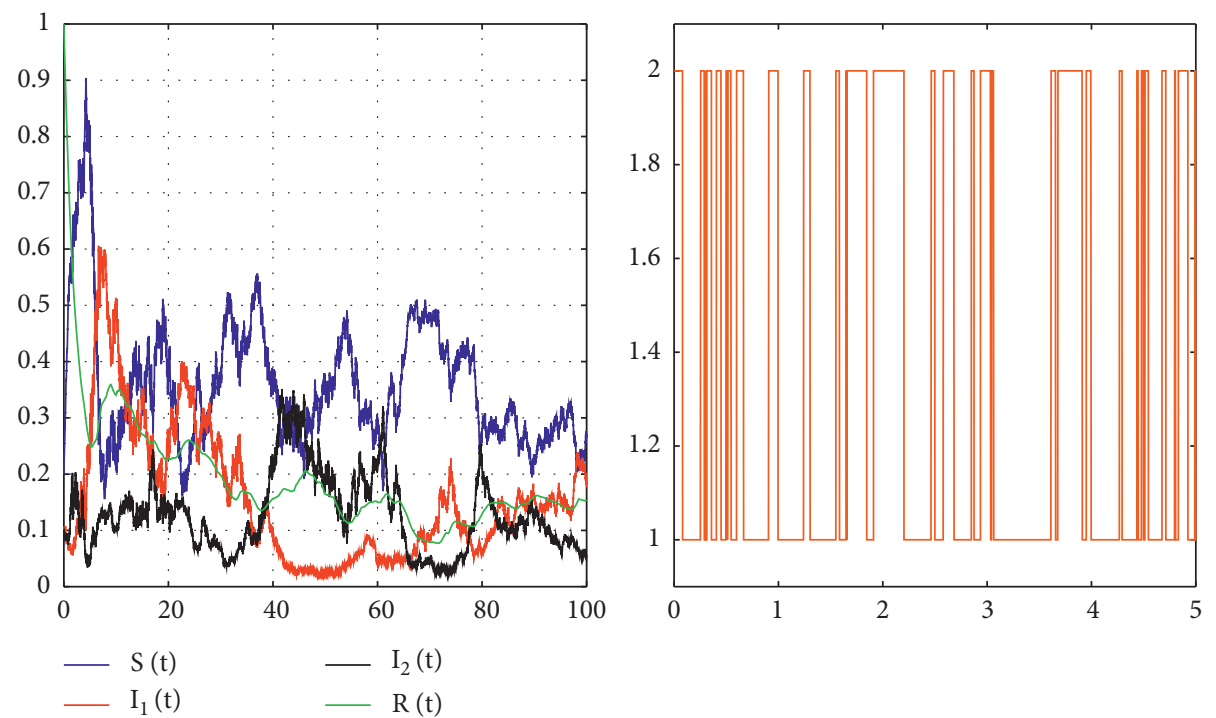

FIgURE 3: Sample paths of $S(t)$ in blue, $I_{1}(t)$ in red, $I_{2}(t)$ in black, $R(t)$ in green, and $r(t)$ in brown in Example 1. 


$$
\liminf _{t \rightarrow \infty}\langle 1-S\rangle_{t} \geq\left(\frac{\check{\mu}+\check{\delta}+\min \left\{\widehat{\gamma}_{1}, \widehat{\gamma}_{2}\right\}}{\check{\mu}+\check{\delta}}\right) I^{*} .
$$

This completes the proof.

\section{Simulation}

In the following, we give numerical simulations to illustrate the main theoretical results.

Example 1. We consider the Markov chain $(r(t))_{t \geq 0}$ taking values in $\mathbb{S}=\{1,2\}$, with generator defined by the following:

$$
\Phi=\left(\begin{array}{cc}
-1 & 1 \\
2 & -2
\end{array}\right)
$$

The parameter values of (4) are presented in Table 1.

Exact computations supply us $\sum_{i=1}^{2} \pi_{i} D_{j}=-0.0352$ and $\sum_{i=1}^{2} \pi_{i} A_{j}=-0.0467$. Consequently, by Theorem 2 , the disease-free equilibrium $E_{0}$ is globally stochastically asymptotically stable on $\mathbb{D}$. Figure 2 confirms this result.

Example 2. In this example, we use the parameter values given in Table 2 and the Markov chain used in Example 1. By simply computing, we obtain $R_{0}^{s}>0$. Theorem 4 shows that the two epidemics persist in the population. Figure 3 confirms this result.

\section{Conclusion}

This paper investigates a stochastic switched epidemic model with double epidemic diseases and cure rate affected with a composite of environmental perturbations modeled by white and color noises. We established conditions for extinction and persistence of diseases.

The theoretical result given in this paper shows that the stationary distribution of Markov chain $r(t)$ controls the propagation of the epidemic in the population. To know, if the Markov chain $r(t)$ takes the values which lead to find that $\sum_{i=1}^{m} \pi_{i} D_{i}<0$ and $\sum_{i=1}^{m} \pi_{i} A_{i}<0$, then the epidemic diseases die out. We have given numerical simulations in order to support and illustrate the main results of this paper.

In this perspective, we prepare to include Lévy noises into epidemic models (4) to describe the effect of sudden and dangerous phenomena such as pandemic, tsunami, and volcano.

\section{Data Availability}

The data were curated by the authors and are available upon request.

\section{Conflicts of Interest}

The authors declare that they have no conflicts of interest.

\section{References}

[1] R. M. Anderson, B. Anderson, and R. M. May, Infectious Diseases of Humans: Dynamics and Control, Oxford University Press, Oxford, UK, 1992.

[2] C. V. De-Leon, "On the global stability of SIS, SIR and SIRS epidemic models with standard incidence," Chaos, Solitons \& Fractals, vol. 44, no. 12, pp. 1106-1110, 2011.

[3] X. Jin, S. Jin, and D. Gao, "Mathematical analysis of the rossMacDonald model with quarantine," Bulletin of Mathematical Biology, vol. 82, no. 4, 2020.

[4] C. Tian, Q. Zhang, and L. Zhang, "Global stability in a networked SIR epidemic model," Applied Mathematics Letters, vol. 107, Article ID 106444, 2020.

[5] L. Zhao, Z.-C. Wang, and S. Ruan, "Dynamics of a timeperiodic two-strain SIS epidemic model with diffusion and latent period," Nonlinear Analysis: Real World Applications, vol. 51, Article ID 102966, 2020.

[6] R. K. Upadhyay, A. K. Pal, S. Kumari, and P. Roy, "Dynamics of an SEIR epidemic model with nonlinear incidence and treatment rates," Nonlinear Dynamics, vol. 96, no. 4, pp. 2351-2368, 2019.

[7] W. O. Kermack and A. G. McKendrick, "A contribution to the mathematical theory of epidemics," Proceedings of the Royal Society of London. Series A, Containing Papers of a Mathematical and Physical Character, vol. 115, no. 772, pp. 700-721, 1927.

[8] L. Xiang, Y. Zhang, and J. Huang, "Stability analysis of a discrete SIRS epidemic model with vaccination," Journal of Difference Equations and Applications, vol. 26, no. 3, pp. 309-327, 2020.

[9] M. Lu, J. Huang, S. Ruan, and P. Yu, "Bifurcation analysis of an SIRS epidemic model with a generalized nonmonotone and saturated incidence rate," Journal of Differential Equations, vol. 267, no. 3, pp. 1859-1898, 2019.

[10] S. Han, C. Lei, and X. Zhang, "Qualitative analysis on a diffusive SIRS epidemic model with standard incidence infection mechanism," Zeitschrift für angewandte Mathematik und Physik, vol. 71, no. 6, pp. 1-23, 2020.

[11] X.-z. Meng, "Stability of a novel stochastic epidemic model with double epidemic hypothesis," Applied Mathematics and Computation, vol. 217, no. 2, pp. 506-515, 2010.

[12] X. Meng, S. Zhao, T. Feng, and T. Zhang, "Dynamics of a novel nonlinear stochastic SIS epidemic model with double epidemic hypothesis," Journal of Mathematical Analysis and Applications, vol. 433, no. 1, pp. 227-242, 2016.

[13] A. Miao, X. Wang, T. Zhang, W. Wang, and B. S. A. Pradeep, "Dynamical analysis of a stochastic SIS epidemic model with nonlinear incidence rate and double epidemic hypothesis," Advances in Difference Equations, vol. 2017, no. 1, Article ID 226, 2017.

[14] A. El Koufi, M. Naim, A. Bennar, and N. Yousfi, "Stability analysis of a stochastic SIS model with double epidemic hypothesis and specific nonlinear incidence rate," Communications in Mathematical Biology and Neuroscience, vol. 2018, 2018.

[15] J. Zhao, L. Wang, and Z. Han, "Stability analysis of two new SIRS models with two viruses," International Journal of Computer Mathematics, vol. 95, no. 10, pp. 2026-2035, 2018.

[16] X. B. Zhang, H. F. Huo, H. Xiang, Q. Shi, and D. Li, "The threshold of a stochastic SIQS epidemic model," Physica A: Statistical Mechanics and Its Applications, vol. 482, pp. 362374, 2014. 
[17] A. El Koufi, J. Adnani, A. Bennar, and N. Yousfi, "Analysis of a stochastic SIR model with vaccination and nonlinear incidence rate," International Journal of Differential Equations, vol. 2019, Article ID 9275051, 9 pages, 2019.

[18] Y. Chen, B. Wen, and Z. Teng, "The global dynamics for a stochastic SIS epidemic model with isolation," Physica A: Statistical Mechanics and Its Applications, vol. 492, pp. 1604-1624, 2018.

[19] M. El Fatini, A. Lahrouz, R. Pettersson, A. Settati, and R. Taki, "Stochastic stability and instability of an epidemic model with relapse," Applied Mathematics and Computation, vol. 316, pp. 326-341, 2018.

[20] Q. Liu, D. Jiang, T. Hayat, A. Alsaedi, and B. Ahmad, "Dynamical behavior of a higher order stochastically perturbed SIRI epidemic model with relapse and media coverage," Chaos, Solitons \& Fractals, vol. 139, Article ID 110013, 2020.

[21] T. Tang, Z. Teng, and Z. Li, "Threshold behavior in a class of stochastic SIRS epidemic models with nonlinear incidence," Stochastic Analysis and Applications, vol. 33, no. 6, pp. 9941019, 2015.

[22] H. Qi, L. Liu, and X. Meng, "Dynamics of a nonautonomous stochastic sis epidemic model with double epidemic hypothesis," Complexity, vol. 2017, Article ID 4861391, 14 pages, 2017.

[23] S. P. Rajasekar and M. Pitchaimani, "Qualitative analysis of stochastically perturbed SIRS epidemic model with two viruses," Chaos, Solitons \& Fractals, vol. 118, pp. 207-221, 2019.

[24] M. Slatkin, "The dynamics of a population in a Markovian environment," Ecology, vol. 59, no. 2, pp. 249-256, 1978.

[25] M. Liu, X. He, and J. Yu, "Dynamics of a stochastic regimeswitching predator-prey model with harvesting and distributed delays," Nonlinear Analysis: Hybrid Systems, vol. 28, pp. 87-104, 2018.

[26] X. Yu, S. Yuan, and T. Zhang, "Persistence and ergodicity of a stochastic single species model with Allee effect under regime switching," Communications in Nonlinear Science and Numerical Simulation, vol. 59, pp. 359-374, 2018.

[27] X. Mao and C. Yuan, Stochastic Differential Equations with Markovian Switching, Imperial College Press, London, UK, 2006.

[28] X. Zhang and H. Peng, "Stationary distribution of a stochastic cholera epidemic model with vaccination under regime switching," Applied Mathematics Letters, vol. 102, Article ID 106095, 2020.

[29] S. Wang, Y. Peng, and F. Wang, "Stability and asymptotic behavior of a regime-switching SIRS model with beddingtonDeAngelis incidence rate," Mathematical Problems in Engineering, vol. 2020, Article ID 7181939, 12 pages, 2020.

[30] X. Li, X. Li, and Q. Zhang, "Time to extinction and stationary distribution of a stochastic susceptible-infected-recoveredsusceptible model with vaccination under Markov switching," Mathematical Population Studies, vol. 27, no. 4, pp. 259-274, 2020.

[31] A. El Koufi, A. Bennar, and N. Yousfi, "Dynamics of a stochastic SIRS epidemic model with regime switching and specific functional response," Discrete Dynamics in Nature and Society, vol. 2020, Article ID 5898456, 13 pages, 2020.

[32] X. Mao, G. Marion, and E. Renshaw, "Environmental Brownian noise suppresses explosions in population dynamics," Stochastic Processes and Their Applications, vol. 97, no. 1, pp. 95-110, 2002.

[33] C. Yuan and X. Mao, "Robust stability and controllability of stochastic differential delay equations with Markovian switching," Automatica, vol. 40, no. 3, pp. 343-354, 2004. 